\title{
An interaural electrode pairing clinical research system for binaural cochlear implants
}

\section{Hu, Hongmei}

IEEE

2014

Hu , H , Ewert , S , Campbell , T , Kollmeier , B \& Dietz , M 2014 , An interaural electrode pairing clinical research system for binaural cochlear implants . in 2014 IEEE China Summit and International Conference on Signal and Information Processing (Chinasip) . IEEE , Xi'an , pp. 66-70, International conference on signal and information processing , Xi'an , China , 09/07/2014 . https://doi.org/10.1109/ChinaSIP.2014.6889203

http://hdl.handle.net/10138/161135

https://doi.org/10.1109/ChinaSIP.2014.6889203

publishedVersion

Downloaded from Helda, University of Helsinki institutional repository.

This is an electronic reprint of the original article.

This reprint may differ from the original in pagination and typographic detail.

Please cite the original version. 


\title{
AN INTERAURAL ELECTRODE PAIRING CLINICAL RESEARCH SYSTEM FOR BILATERAL COCHLEAR IMPLANTS
}

\author{
Hongmei Hu, Stephan Ewert, Tom Campbell, Birger Kollmeier, Mathias Dietz \\ Medizinische Physik, Universität Oldenburg and Cluster of Excellence "Hearing4all”, Germany
}

\begin{abstract}
Bilateral cochlear implants (BiCIs) have succeeded in improving the spatial hearing performance of bilateral CI users, albeit with considerable variability across implantees. Limited success can be caused by an interaural mismatch of the place-of-stimulation that arises from electrode arrays being inserted at different depths in each cochlea. In comparison to subjective pairing methods such as pitch matching, one promising objective measure based on electrically evoked auditory brainstem responses (EABR), the binaural interaction component (BIC), could be used to optimize the interaural electrode pairing (IEP) in BiCIs. Matched interaural electrodes are expected to facilitate binaural functions such as binaural fusion, localization, or detection of signals in noise. An IEP system, currently under development for clinical research, is proposed. The system offers subjective and objective IEP methods for BiCI: a psychoacoustic test module for pitch ranking and interaural pulse time difference (IPTD) sensitivity, and a binaural and monaural EABR recording module to derive the BIC. Psychoacoustic and IEP measures from two implantees are presented.
\end{abstract}

Index Terms - Bilateral cochlear implants, EEG, Binaural interaction components, Interaural electrode pairing, Objective measures

\section{INTRODUCTION}

Bilateral cochlear implantation has sought to restore the advantages of binaural hearing to the deaf by providing binaural cues normally important for binaural perception. Most bilateral cochlear implant (BiCI) users have shown improvements in sound localization compared to their ability when using only one CI. However, compared to normal hearing $(\mathrm{NH})$ individuals, localization accuracy of BiCI users has still been much worse and there has remained a large variability in performance amongst CI users [1-4]. One reason identified for the difference in performance between $\mathrm{CI}$ and $\mathrm{NH}$ subjects is that the inputs to the $\mathrm{NH}$ binaural system from the two ears can be assumed to be well matched, whilst in CI users the peripheral auditory inputs to the binaural system at the two ears require appropriate matching strategies, e.g. for interaural electrodes.

Several approaches have been used to define the best-matched interaural electrode pairs for enabling binaural function for bilateral CI users so that binaural fusion, localization and detection of signals in noise could be maximized. The approaches include the clinically used computed tomography scans that match electrodes based on insertion depth [5-7] and interaural pitch comparisons to aid interaural electrode pairing [8-15]). Electrically evoked auditory brain-

This work was funded by European Union under the Advancing Binaural Cochlear Implant Technology (ABCIT) grant agreement (No. 304912). The authors would like to thank Stefan Strahl (MED-EL) for RIB II support and the participating CI users. (e-mail: hongmei.hu@uni-oldenburg.de). stem response (EABR) measurements have emerged as a promising method for assigning best-matched interaural electrode pairs. EABR could potentially replace psychophysical approaches, especially for pediatric patients. The hypothesis has been that, when two implants are put into place, interaurally corresponding electrodes stimulate auditory-nerve fibers from comparable cochlear regions in the two ears. Accordingly, stimulation of such comparable pairs of electrodes would cause an enhanced binaural interaction relative to stimulation of non-comparable pairs $[16,17]$. He et al. corroborated this hypothesis in CI users [13], demonstrating that the binaural interaction component (BIC) of the EABR could be used to match electrodes in bilateral CI users.

The aim of the current study was to establish a clinical research system including subjective psychophysical listening tests and objective EABR recordings, to support an optimal interaural electrode pairing (IEP) strategy in bilateral CI users. The IEP experiment system was developed in MATLAB (Mathworks) for fast psychoacoustic testing and EABR recording. The system includes psychoacoustic pre-testing modules (e.g. loudness balancing), a pitch ranking module, an interaural pulse time difference (IPTD) sensitivitytesting module, and a binaural and monaural EABR module.

The paper is organized as follows: after introduction of the BIC in BiCI users in Section 2, the IEP experiment system is described in Section 3. The results from two BiCI adults are presented in Section 4 , followed by the conclusion in Section 5 .

\section{BIC IN BILATERAL COCHLEAR IMPLANTEES}

Binaural interaction has been demonstrated to occur in the subcortical auditory system within the dorsal cochlear nucleus, superior olivary nucleus, dorsal nucleus of the lateral nucleus, and the inferior colliculus [18]. In evoked response studies, binaural processing is assessed by the binaural interaction component. The BIC can be isolated from the auditory brainstem response (ABR), an evoked response in the electroencephalogram (EEG) that is generated by subcortical structures. The BIC is defined as the difference between the potential obtained with binaural stimulation $(B)$ and the sum of the potentials obtained with monaural stimulation $(L+R)$, symbolically $B I C=B-(L+R) \underline{[19]}$. Any significant deviation from BIC $=0$ is understood to indicate a non-linear additivity, i.e. a functional coupling of left and right signals. During the past 40 years, acoustically stimulated BIC has been extensively investigated [19-21].

More recently, electrophysiological evidence of binaural interaction has also been revealed in bilateral CI users [13,17,22]. These responses evoked by direct stimulation of cochlear-implant electrodes rather than acoustical stimulation have been termed EABR, and exhibit distinguishing characteristics from ABRs [18,21], namely earlier peak latencies and higher peak amplitudes [23-25]. A pronounced electrically evoked BIC, consisting of a negative peak around $3.6 \mathrm{~ms}$ followed by a positive peak near $4.4 \mathrm{~ms}$ was shown 


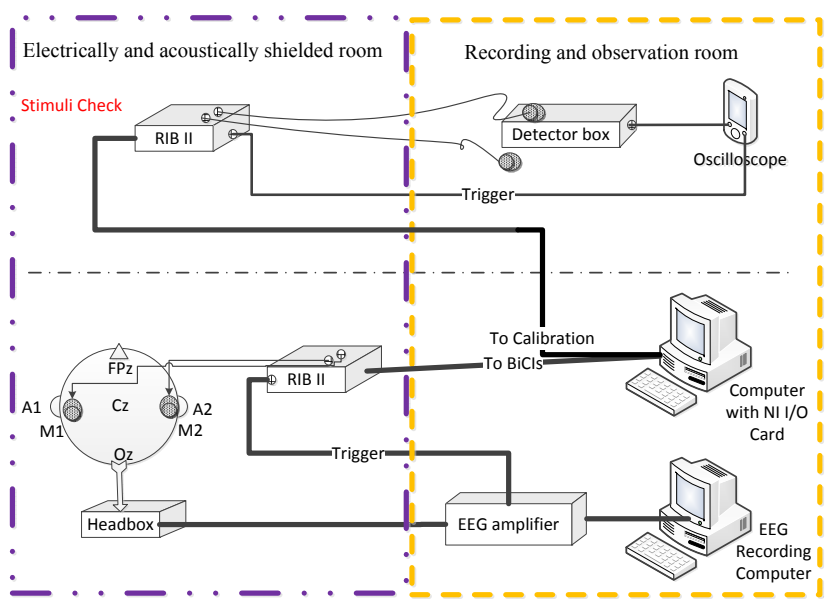

Fig. 1. The IEP clinical research system consists of sub-systems for CI stimulation, and EEG recording. The upper part is calibration

in [16], being attributable to the fact that electrical pulses stimulated fibers of the auditory nerve synchronously. Accordingly, the established nature of the BIC, together with the high amplitude of the electrically evoked BIC motivates a further investigation of the electrically evoked BIC as a method for pairing the positions of intracochlear electrodes $\underline{[13,16] \text {. }}$.

\section{INTERAURAL ELECTRODE PAIRING CLINICAL RESEARCH SYSTEM}

The proposed IEP clinical research system is comprised of psychophysical test procedures and an EEG procedure. Five monaural and binaural (bilateral) psychophysical test procedures are included: loudness estimation, loudness balancing, interaural pitch ranking, sound image centering and IPTD sensitivity. The loudness estimation procedure estimates the maximum comfortable level (MCL) and hearing threshold (HL); the loudness balancing procedure determines levels of equal loudness for each electrode pair; the pitch ranking procedure identifies the pitch-matched electrodes; the sound image centering is a pre-test and interaural level calibration for the IPTD sensitivity procedure; and the IPTD procedure determines the binaurally most sensitive electrode pair. EEG recordings then derive scalp-measured EABRs, with the presentation level obtained from the loudness balancing procedure. The IEP obtained by pitch ranking and the IEP derived from the BIC tuning can then be compared against the IEP drawn from IPTD sensitivity, which is defined as the standard reference.

\subsection{Equipment and stimuli}

The stimulation and EEG setup for the current BiCIs IEP clinical research system is schematized in Fig. 1. A research interface box (RIB II, manufactured at University of Innsbruck, Austria) delivers monaural or bilaterally synchronized electrical pulses directly to the MED-EL CIs. Prior to the experiment, the stimuli are verified using a detector box (a MED-EL CI simulator). A user-friendly graphical user interface (GUI) as shown in Fig. 2 (a), controls the electrical stimulation of the IEP clinical research system via a stimulation computer with a National Instruments I/O card.

Most of the psychophysical tests are based on standardized procedures employed in previous BiCI studies [13, 17, 22, 26]. For

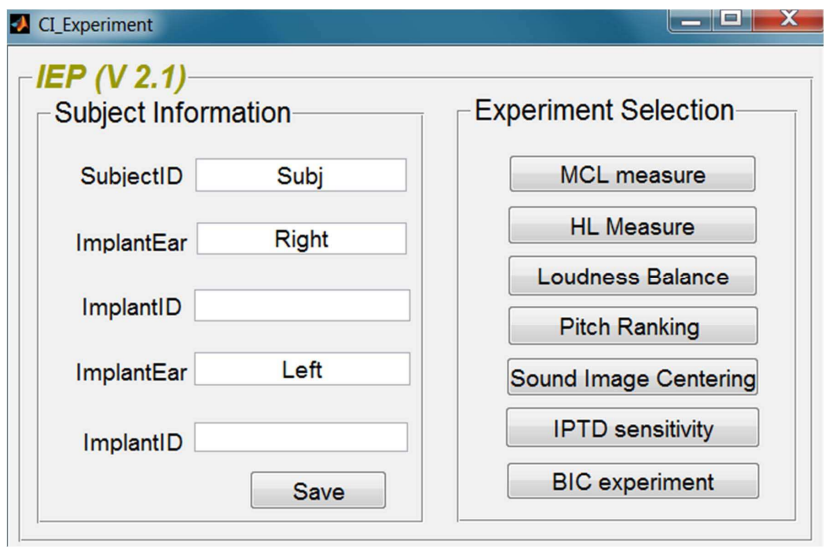

(a)

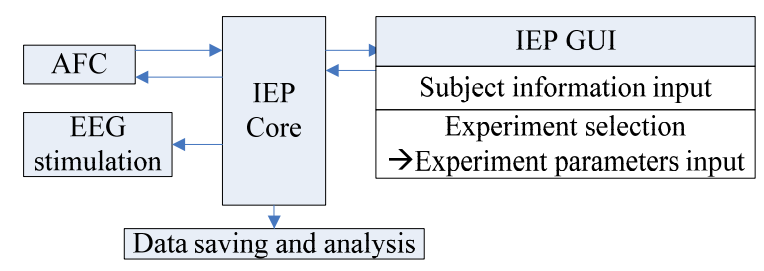

(b)

Fig. 2. The IEP CI experiment GUI is shown in panel (a), with participant information to be entered on the left, and experiment selection on the right. The experiment selection invokes the AFC and EABR recording modules as indicated by the schematic outline of the software in panel (b).

the psychophysical testing the freely available Oldenburg AFC software [27] which was extended here to operate the RIB II interface, is invoked by the IEP GUI. For potential future extension, all the AFC experiments already implemented for acoustical stimulation in [27] can be used for direct electrode stimulation via RIB II. Figure 2 (b) shows a schematic diagram of the IEP clinical research system's software. The GUI offers a user-friendly way to enter patient information, for parameter selection (e.g. reference CI and electrode), and for starting the test modules.

In the following system evaluation, the reference CI was left and the reference electrode was electrode number 5, referred to as L5. Eight electrodes in the contralateral CI (right) were selected (R1R6, R9, R12). There were thus 8 electrode pairs, namely [L5, R1], [L5, R2], [L5, R3], [L5, R4], [L5, R5], [L5, R6], [L5, R9], [L5, R12]. The stimulus was a train of charge-balanced biphasic pulses, with $60 \mu$ s phase duration, and $2.1 \mu$ s interphase gap presented via monopolar stimulation mode. All stimuli for these experiments were constant amplitude pulse trains presented at a rate of 19.9 pulses per second (pps), either to a single electrode or to an interaural pair of electrodes. The rate of pulsatile stimulation was lower than typical stimulation rates used in clinical CI processors but optimal for the assessment of BIC [13]. Moreover, IPTD perception is improved at low stimulation rates [28]. The participants' responses during the psychophysical testing were obtained using a touch screen monitor connected to the stimulation computer. For psychophysical testing, 400-ms pulse trains (i.e. a train of 8 pulses) were used, while for EEG, the electrical pulse train was presented continuously. 


\subsection{Psychophysical tests}

Tests are performed in the following order: Loudness estimates obtain the participant's dynamic range (DR) by measuring the maximum comfortable level and the hearing threshold. The loudness balancing procedure is performed for each interaural electrode pair. The stimulation levels judged by the participants to be equally loud are saved and used in the pitch ranking and BIC recording procedures, respectively. The sound image centering is a pre-testing procedure for IPTD sensitivity testing, in which the participants are instructed to adjust the presented level at one ear to perceive a centralized sound image. The IPTD sensitivity experiment includes two steps: the individual IPTD threshold estimation in one electrode pair for each participant, and the IPTD sensitivity experiment with the estimated individual IPTD threshold for all the selected electrode pairs. Each participant receives detailed written instructions before each specific experiment. The participant then responds to stimulation by pressing different buttons on the touch screen as instructed for the specific procedures.

\subsubsection{Loudness estimation}

Each reference electrode is stimulated individually, and a simple loudness adjustment procedure employing a touch screen is used to determine the MCL and HL. In the MCL testing, the stimulation level is set initially to each CI participant's clinical comfortable level. The participants were instructed to respond via the touch screen on the experiment GUI by pressing 'increase' or 'decrease' buttons to change the present sound levels, and to press the 'OK' button when the stimulus was 'very loud but still comfortable'. A factor of 1.2 is initially used to multiply (for increase) or to divide the stimulation current for adjustment and reduced to 1.05 after the first change of direction during the adjustment. The final presentation level is then stored as MCL. Once the MCL is determined, the participant is asked to adjust the stimulation level to be 'just audible' following the same procedure. The resulting level is then saved as HL. This MCL and HL procedure is repeated three times for each electrode. The dynamic range to current unit mapping is then calculated from the median MCL and HL.

\subsubsection{Loudness balance}

Interaurally loudness-balanced levels for each electrode pair (i.e. 8 pairs) are determined by an analogous adjustment procedure to that used for loudness estimation. The members of each electrode pair are stimulated in two successive intervals in random order. The stimulus presented to the reference CI electrode is fixed in level $(60 \%$ of DR). The same pulse train stimulus is then presented to one of the test CI electrodes contralaterally (e.g. R3). The participant then indicates which of the two stimulus bursts was louder. The procedure adjusts the level with a factor of 1.2 , which is then reduced to 1.05 after the first reversal of adjustment direction. The participant terminates the procedure by pressing the 'OK' button once the stimuli in both ears are perceived as equally loud. The number of repetitions of the measurement for each electrode pair can be adjusted and the average across repetitions is then calculated as final result.

\subsubsection{Interaural pitch ranking}

Members of each interaural electrode pair are directly compared for perceived pitch, using a constant stimulus procedure. Each of these electrode pair members are stimulated in a random order. The participants are asked to indicate in which interval the higher pitch is perceived. The participants are instructed to focus on pitch rather than timbre or loudness.

Here, a selected reference electrode (L5) was paired with 8 target electrodes and each pair was presented ten times, yielding 80 judgments. This experiment was repeated 5 times per reference electrode, yielding 50 responses per electrode pair. Electrode pairs with an average discriminability across 50 repetitions within the range of chance (50 $\pm 14 \%$, corresponding to the $95 \%$ confidence interval) were considered as pitch-matched. For participants with more than one pitch-matched electrode pair, the pair at the medial tonotopic position was chosen. The results of all 8 electrode pairs were calculated and stored.

\subsubsection{Sound image centering}

A lateralization task is performed on each electrode pair to ensure that a single, fused auditory image is perceived in the center of the head. The members of each electrode pair are stimulated simultaneously with zero IPTD.

Here, the reference electrode (L5) had a fixed level (60\% of DR) and the presentation level of the contralateral target electrode was adjusted in level according to the participant's response. After each bilateral stimulation (one interval) the participant selected one of four response alternatives ('left of the center', 'right of the center', 'exactly from the center', or 'other') according to their perception of the sound image. The 'other' button was used in the following cases: more than one sound source was perceived; there was only one sound image, but it could not be adjusted to be centralized after considerable time; the sound could not be fused [15]. Only those electrode pairs for which the participants terminated the experiment with responding 'exactly from the center' are selected for further IPTD sensitivity testing in the next experimental procedure.

\subsubsection{IPTD sensitivity testing}

The goal of this procedure is to systematically investigate the effects of interaural electrode pairing on IPTD discrimination. The member$\mathrm{s}$ of each electrode pair that yield a centralized fused sound image in the previous experiment are stimulated simultaneously with the presentation level on the reference electrode (L5) at a fixed level $(60 \%$ of DR) and the presentation level of the electrodes on the other ear resulting from the sound image centering. A series of measurements using the constant stimulus procedure is used.

Here, each presentation consisted of two 500-ms intervals separated by a $300-m s$ pause. For a fixed IPTD, in one randomly selected interval a left-leading IPTD of IPTD/2 was introduced while the other interval carried a right-leading IPTD of IPTD/2. The participant was required to indicate whether the stimulus in the second interval was perceived from a lateral position either to the left or the right of the first interval.

First, an IPTD of $700 \mu$ s was chosen and 20 two-interval trials were presented for the electrode pair, i.e. [L5, R5]. If the correctly identified lateral positions were between 65 to $85 \%$, the IPTD was defined as threshold IPTD. If the correct rate was lower or higher, the IPTD was increased or decreased respectively and the experiment was repeated until a threshold IPTD was found.

The final IPTD sensitivity experiment was performed using the above determined threshold IPTD and the same procedure for all electrode pairs under investigation, i.e. 8 pairs here. The threshold IPTD from the pre-test ensures performance well above chance but below ceiling performance, independent of the participant's individual sensitivity. Each electrode pair was stimulated ten times in 


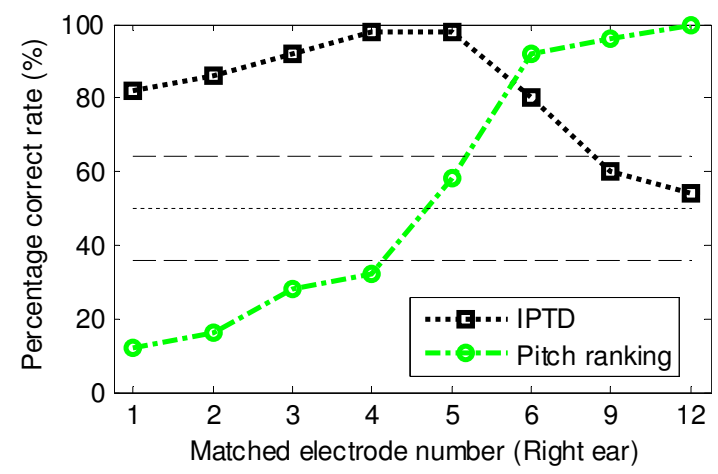

Fig. 3. Results of pitch ranking and IPTD sensitivity procedures for the 8 selected electrode pairs. The electrode pairs within the range of the chance level ( $50 \pm 14 \%$, dashed lines) were considered as pitchmatched, in this case [L5, R5]. IPTD performance was best for [L5, R4] and [L5, R5].

a random order. The experiment was repeated 5 times, yielding 50 repetitions for each electrode pair. The correct rate was determined for all 8 electrode pairs.

\subsection{EEG recording and analysis}

EEG is differentially recorded from $\mathrm{Ag} / \mathrm{AgCl}$ electrodes of the international 10-20 system [29]. In the data shown here, a single $\mathrm{Ag} / \mathrm{AgCl}$ electrode at $\mathrm{A} 1$ was recorded, with an electrode at $\mathrm{FPz}$ serving as the ground, and $\mathrm{Cz}$ as the physical reference. The participant was seated in a recliner and watched a silent subtitled movie within an electrically shielded sound-attenuating booth. EEG is preamplified with a gain of 150 by a headbox and, in turn, amplified in a DC-coupled manner with a gain of 5000. An analog second-order lowpass with a $3-\mathrm{kHz}$ corner frequency is applied by the DSP card of a Synamps 5083 (Neuroscan, Hampstead, New Hampshire, USA), and the signal is digitized at $50 \mathrm{kHz}$ to 16-bit resolution by the A/D convertor of the Synamps system. EABRs evoked by monaural and bilateral stimulation are derived by epoching the EEG from -25 to $30 \mathrm{~ms}$ post-stimulus onset online. Epochs are averaged online for each bilateral interaural electrode pair separately and for each monaural electrode separately, and these separate averages are then corrected offline such that EABR amplitudes are relative to a 1.000 ms prestimulus baseline prior to the $\mathrm{CI}$ artifact that is caused by the biphasic CI pulse. Electrically evoked BICs are then derived offline.

\section{EVALUATION OF THE IEP SYSTEM}

The IEP clinical research system was evaluated with two cochlear implantees with postlingual onset of bilateral severe-to-profound sensorineural hearing loss and MED-EL implant systems. Voluntary informed written consent was obtained with the approval of the Ethics Committee of the University of Oldenburg.

The results of pitch ranking and IPTD sensitivity procedures for the 8 selected electrode pairs of 1 subject's are depicted in Fig. 3 as a function of the electrode number. For the pitch ranking, values below the chance level indicate a lower pitch at the test electrode compared to the reference electrode, while values above chance level indicate a higher pitch percept at the test electrode side. The electrode pairs within the range of the chance level ( $50 \pm 14 \%$, indicated

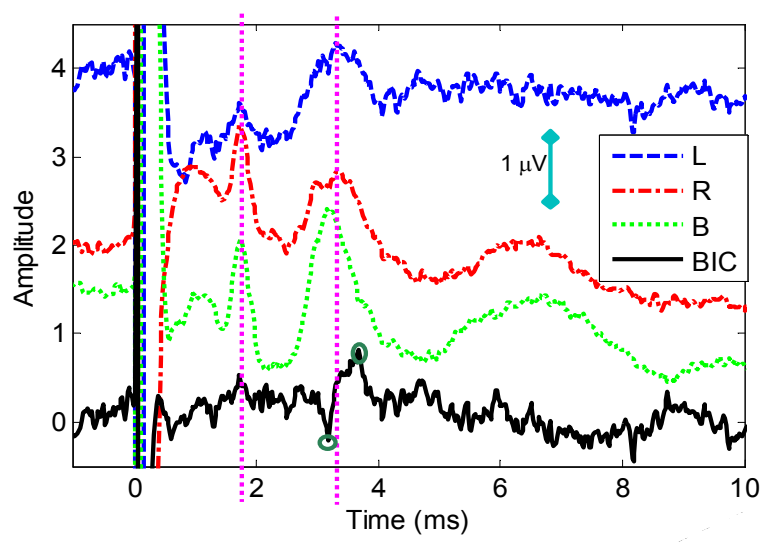

Fig. 4. EABRs and the BIC results for one exemplary electrode pair: EABRs of left CI stimulated only, right CI stimulated only, both CI stimulated simultaneously and the BIC. The y-axis is their amplitude values in $\mu \mathrm{V}$, with additive offsets of $4,2,1.5$ and $0.1 \mu \mathrm{V}$ respectively. The electric artifact can be seen at $0 \mathrm{~ms}$, while wave eIII and $\mathrm{eV}$ are visible at approximately 1.7 and $3.3 \mathrm{~ms}$, respectively.

by the dashed horizontal lines) were considered as pitch-matched, for this case [L5, R5]. The IPTD performance is indicated by the black squares and was highest for the two electrode pairs [L5, R4] and [L5, R5]. High performance in the IPTD task coincided with pitch matching.

The left and right monaural EABRs, the bilateral EABR and the BIC for one electrode pair, [L5, R3], are shown in Fig. 4. For illustration purpose, vertical offsets of $4,2,1.5$, and $0.1 \mu \mathrm{V}$ were added to the four traces from top to bottom, respectively. The BIC response consisted of a negative peak at $3.3 \mathrm{~ms}$ followed by a positive peak around $4 \mathrm{~ms}$, as commensurate with the literature $[13,16]$.

\section{SUMMARY AND CONCLUSIONS}

The typically observed large variability of performance in Binaural CIs is partially attributable to a mismatch in the place-of-stimulation arising from electrode arrays being inserted at different depths in each cochlea. Here, a clinical research system for interaural electrode pairing (IEP) in BiCIs is designed and evaluated. A userfriendly GUI for the IEP experiment system controls an existing psychophysical measurement system (AFC [27]) embedded in the IEP system which was enhanced by an interface module to the MED-EL RIB II. The IEP experiment system includes several state-of-the-art psychophysical IEP methods, including pitch ranking, IPTD sensitivity and a binaural and monaural EABR recording module for BIC measurements.

Evaluation with two adult bilateral CI participants demonstrated the system's functionality and plausible results for the psychophysical IEP methods and a clear BIC were obtained. In the future, more implantees will be recruited and tested with the IEP system. Results of the different electrode pairing methods will be compared to find relations between different pairing methods and for developing an improved interaural electrode pairing strategy for BiCI users. The refined IEP clinical research system is planned to be made available for research and non-commercial applications. 


\section{REFERENCES}

[1] D. W. Grantham, D. H. Ashmead, T. A. Ricketts, R. F. Labadie, and D. S. Haynes, "Horizontal-plane localization of noise and speech signals by postlingually deafened adults fitted with bilateral cochlear implants," Ear Hear, vol. 28, no. 4, pp. 524541, Aug 2007.

[2] R. Y. Litovsky, A. Parkinson, and J. Arcaroli, "Spatial hearing and speech intelligibility in bilateral cochlear implant users," Ear Hear, vol. 30, no. 4, pp. 419-431, Aug 2009.

[3] P. Majdak, M. J. Goupell, and B. Laback, "Two-dimensional localization of virtual sound sources in cochlear-implant listeners," Ear Hear, vol. 32, no. 2, pp. 198-208, Apr 2011.

[4] G. Vecchiato, J. Toppi, L. Astolfi, D. Mattia, P. Malerba, A. Scorpecci, P. Marsella, and F. Ba biloni, "Investigation on the pleasantness of music perception in monolateral and bilateral cochlear implant users by using neuroelectrical source imaging: A pilot study," in EMBC2011, Aug 2011, pp. 8110-8113.

[5] M. A. Marsh, J. Xu, P. J. Blamey, L. A. Whitford, S. A. Xu, J. M. Silverman, and G. M. Clark, "Radiologic evaluation of multichannel intracochlear implant insertion depth," Am J Otol, vol. 14, no. 4, pp. 386-391, Jul 1993.

[6] J. Xu, S. A. Xu, L. T. Cohen, and G. M. Clark, "Cochlear view: postoperative radiography for cochlear implantation," Am J Otol, vol. 21, no. 1, pp. 49-56, Jan 2000.

[7] M. W. Skinner, D. R. Ketten, L. K. Holden, G. W. Harding, P. G. Smith, G. A. Gates, J. G. Neely, G. R. Kletzker, B. Brunsden, and B. Blocker, "CT-derived estimation of cochlear morphology and electrode array position in relation to word recognition in Nucleus-22 recipients," J Assoc Res Otolaryngol, vol. 3, no. 3, pp. 332-350, Sep 2002.

[8] R. J. van Hoesel and G. M. Clark, "Psychophysical studies with two binaural cochlear implant subjects," J Acoust Soc Am, vol. 102, no. 1, pp. 495-507, Jul 1997.

[9] C. J. Long, D. K. Eddington, H. S. Colburn, and W. M. Rabinowitz, "Binaural sensitivity as a function of interaural electrode position with a bilateral cochlear implant user," J Acoust Soc Am, vol. 114, no. 3, pp. 1565-1574, Sep 2003.

[10] R. J. van Hoesel and R. S. Tyler, "Speech perception, localization, and lateralization with bilateral cochlear implants," $J$ Acoust Soc Am, vol. 113, no. 3, pp. 1617-1630, Mar 2003.

[11] R. J. van Hoesel, "Exploring the benefits of bilateral cochlear implants," Audiol Neurootol, vol. 9, no. 4, pp. 234-246, Aug 2004.

[12] R. J. van Hoesel, "Sensitivity to binaural timing in bilateral cochlear implant users," J Acoust Soc Am, vol. 121, no. 4, pp. 2192-2206, Apr 2007.

[13] S. He, C. J. Brown, and P. J. Abbas, "Effects of stimulation level and electrode pairing on the binaural interaction component of the electrically evoked auditory brain stem response," Ear Hear, vol. 31, no. 4, pp. 457-470, Aug 2010.

[14] M. J. Goupell, C. Stoelb, A. Kan, and R. Y. Litovsky, "Effect of mismatched place-of-stimulation on the salience of binaural cues in conditions that simulate bilateral cochlear-implant listening," J Acoust Soc Am, vol. 133, no. 4, pp. 2272-2287, Apr 2013.
[15] A. Kan, C. Stoelb, R. Y. Litovsky, and M. J. Goupell, "Effect of mismatched place-of-stimulation on binaural fusion and lateralization in bilateral cochlear-implant users," J Acoust Soc Am, vol. 134, no. 4, pp. 2923-2936, Oct 2013.

[16] M. Pelizzone, A. Kasper, and P. Montandon, "Binaural interaction in a cochlear implant patient," Hear Res, vol. 48, no. 3, pp. 287-290, Oct 1990.

[17] Z. M. Smith and B. Delgutte, "Using evoked potentials to match interaural electrode pairs with bilateral cochlear implants.," J Assoc Res Otolaryngol, vol. 8, no. 1, pp. 134-151, Mar 2007.

[18] R. A. Dobie and S. J. Norton, "Binaural interaction in human auditory evoked potentials," Electroencephalogr Clin Neurophysiol, vol. 49, no. 3-4, pp. 303-313, Aug 1980.

[19] H. Riedel and B. Kollmeier, "Auditory brain stem responses evoked by lateralized clicks: is lateralization extracted in the human brain stem?," Hear Res, vol. 163, no. 1-2, pp. 12-26, Jan 2002.

[20] J. N. Gardi and C. I. Berlin, "Binaural interaction components. Their possible origins in guinea pig auditory brainstem response," Arch Otolaryngol, vol. 107, no. 3, pp. 164-168, Mar 1981.

[21] R. A. Levine, "Binaural interaction in brainstem potentials of human subjects," Annals of Neurology, vol. 9, no. 4, pp. 384393, Sept. 1981.

[22] K. A. Gordon, C. Salloum, G. S. Toor, R. van Hoesel, and B. C. Papsin, "Binaural interactions develop in the auditory brainstem of children who are deaf: effects of place and level of bilateral electrical stimulation," J Neurosci, vol. 32, no. 12, pp. 4212-4223, Mar 2012.

[23] C. van den Honert and P. H. Stypulkowski, "Characterization of the electrically evoked auditory brainstem response (ABR) in cats and humans," Hear Res, vol. 21, no. 2, pp. 109 - 126, Feb 1986.

[24] R. T. Miyamoto and D. D. Brown, "Electrically evoked brainstem responses in cochlear implant recipients," Otolaryngol Head Neck Surg, vol. 96, no. 1, pp. 34-38, Jan 1987.

[25] P. J. Abbas and C. J. Brown, "Electrically evoked brainstem potentials in cochlear implant patients with multi-electrode stimulation," Hear Res, vol. 36, no. 2-3, pp. 153-162, Nov 1988.

[26] P. Majdak, B. Laback, and W. D. Baumgartner, "Effects of interaural time differences in fine structure and envelope on lateral discrimination in electric hearing," J Acoust Soc Am, vol. 120, no. 4, pp. 2190-2201, Oct 2006.

[27] S. D. Ewert, "AFC - a modular framework for running psychoacoustic experiments and computational perception models," in Proceedings of the International Conference on Acoustics (AIA-DAGA 2013), March 18 - March 21 2013, pp. 13261329.

[28] R. J. van Hoesel, G. L. Jones, and R. Y. Litovsky, "Interaural time-delay sensitivity in bilateral cochlear implant users: effects of pulse rate, modulation rate, and place of stimulation," $J$ Assoc Res Otolaryngol, vol. 10, no. 4, pp. 557-567, Dec 2009.

[29] F. Sharbrough, G. E. Chatrian, R. P. Lesser, H. Luders, M. Nuwer, and T. W. Picton, "American Electroencephalographic Society guidelines for standard electrode position nomenclature," J Clin Neurophysiol, vol. 8, pp. 200-202, Apr 1991. 